\title{
AVALIAÇÃO DE TECNOLOGIAS: UMA CARACTERIZAÇÃO DA ÁREA POR MEIO DE REVISÃO SISTEMÁTICA
}

Tiago Paz Lasmar (tplasmar@gmail.com) - Núcleo de Tecnologia da Qualidade e Inovação, Universidade Federal de Minas Gerais (UFMG).

Raoni Barros Bagno (rbagno@dep.ufmg.br) - Núcleo de Tecnologia da Qualidade e Inovação, Universidade Federal de Minas Gerais (UFMG).

\section{RESUMO}

Com o aumento da complexidade, dos desenvolvimentos tecnológicos, surge a necessidade de se coletar e analisar diversas informações com o objetivo de dar suporte à tomada de decisão aos gestores de tecnologias. Neste contexto este processo denominado de avaliação de tecnologia se torna fundamental. Na literatura, existem diversas pesquisas que tem como objeto de estudo a "avaliação de tecnologia", porém, não se tem total clareza em relação à área de pesquisa. O objetivo deste trabalho é, por meio de uma revisão sistemática da literatura, caracterizar as áreas de conhecimento relacionadas à "avaliação de tecnologias" identificando em quais contextos e por quem ela é aplicada e quais são os métodos utilizados. Percebeu-se que os objetivos de se fazer a avaliação foram sendo alterados ao longo do tempo à medida que os interesses das organizações envolvidas também foram mudando. O governo de diferentes países e as empresas são os principais envolvidos no processo, estando à avaliação de tecnologias presente desde o contexto de criação de políticas públicas, até na formulação de estratégias de P\&D.

Palavras chave: avaliação de tecnologias; technology forecasting; technology foresight; technology assessment; revisão sistemática. 


\section{INTRODUÇÃO}

A economia tem passado por uma transformação em que elementos como o conhecimento e o desenvolvimento tecnológico assumem papel de destaque (CASTELLS; HALLS, 1995). Nesse contexto a inovação de base tecnológica, comumente com raízes em ambientes de pesquisa científica, tem sido pauta nos debates sobre aumento de competitividade de diferentes instituições.

À medida que a complexidade, o ritmo e a globalização dos desenvolvimentos tecnológicos continuam a aumentar, os desafios de transformar tecnologias em negócios são multiplicados (PHAAL, 2004).

Dentro de uma perspectiva de gestão de novas tecnologias surge a necessidade de se estabelecer estratégias de apoio aos gestores envolvidos. Avaliar uma tecnologia consiste em coletar e analisar suas diversas informações com o objetivo de dar suporte à tomada de decisão ao longo de alguma das fases do processo de gestão tecnológica.

Segundo Porter et al (2004) as decisões que precisam ser tomadas dizem respeito à definição de prioridades para os esforços de pesquisa e desenvolvimento (P \& D), a compreensão e o gerenciamento dos riscos de inovação tecnológica, a exploração da propriedade intelectual e o aumento da competitividade de produtos, processos e serviços.

Ainda segundo os autores, este processo de avaliação de tecnologias pode ser realizado por diferentes atores em diferentes níveis, desde o ponto de vista mais amplo, de um país até da visão individual de uma empresa. O processo para cada um desses agentes apresenta particularidades como o objetivo da avaliação, tempo de execução, profundidade do estudo, metodologias utilizadas. Entender essas especificidades é necessário para avanços tanto práticos como teóricos dessa temática.

$\mathrm{Na}$ literatura, existem diversas pesquisas que tem como objeto de estudo a "avaliação de tecnologia". Porém, ainda não se tem claro quais são as principais frentes de pesquisa em cada área, bem como os principais termos utilizados para especificar cada frente. Diferentes formas de se referir à avaliação de tecnologias, como "technology assessment", "technology foresight" e "technology forecasting" ganharam destaque em momentos diferentes, e houve relativamente pouco esforço para esclarecer suas semelhanças e diferenças (COATES, VARY, 2004).

A partir desse contexto, o presente trabalho tem por objetivo caracterizar as áreas de conhecimento relacionadas à "avaliação de tecnologias" identificando em quais contextos a 
avaliação é aplicada, quais são os principais objetivos de quem faz a avaliação, quem são os principais agentes envolvidos e quais são os métodos utilizados.

$\mathrm{O}$ artigo segue apresentando a metodologia utilizada, posteriormente os principais resultados obtidos no estudo.

\section{METODOLOGIA}

Para alcançar os objetivos propostos a metodologia utilizada foi a revisão sistemática da literatura. Segundo Kitchenham et al (2004) o processo de revisão sistemática consiste em coletar, conhecer, compreender, analisar, sintetizar e avaliar um conjunto de artigos científicos. Por meio de uma revisão sistemática é possível obter uma avaliação criteriosa e confiável das pesquisas realizadas dentro de uma temática específica (BRERETON et al, 2005). No contexto deste trabalho, o passo inicial para a realização da análise foi a escolha da base de dados de busca dos artigos. Foi escolhida a base do Scopus pelo fato de seus dados poderem ser tratados pelo software RStudio em seu módulo de análise bibliométrica.

$\mathrm{Na}$ sequência foram definidas as palavras-chave e as lógicas de busca para a realização da pesquisa. Foram identificadas previamente algumas variações de palavras chaves, que estão relacionadas ao sentido do termo "avaliação de tecnologias". Os termos foram pesquisados em inglês. Para a coleta dos dados, foram pesquisadas na referida base de dados os termos entre aspas no campo principal de busca. Foi utilizada a estratégia de busca por radicais para conseguir captar pequenas variações terminológicas das palavras.

A Tabela 1 demonstra a síntese dos resultados das pesquisas realizadas, bem como os termos pesquisados e os filtros aplicados.

TABELA 1 - Síntese dos resultados da busca na base de dados.

\begin{tabular}{|c|c|c|c|c|}
\hline Palavra Chave & $\begin{array}{c}\text { Termo } \\
\text { Pesquisado }\end{array}$ & $\begin{array}{l}\text { \# Resultados } \\
\text { encontrados }\end{array}$ & Filtros aplicados & $\begin{array}{c}\text { \# Resultados } \\
\text { finais }\end{array}$ \\
\hline $\begin{array}{l}\text { Technology } \\
\text { Assessment }\end{array}$ & $\begin{array}{l}\text { "technolog* } \\
\text { assess*" or } \\
\text { assess* } \\
\text { technolog*" }\end{array}$ & 4070 & $\begin{array}{c}\text { Document type: } \\
\text { Article Language: } \\
\text { English } \\
\text { Publication } \\
\text { Stage: Final } \\
\text { Search type: } \\
\text { Journal Ano: } 1980 \\
\text { até } 2019\end{array}$ & 1987 \\
\hline $\begin{array}{l}\text { Technology } \\
\text { Forecasting }\end{array}$ & $\begin{array}{l}\text { "technolog* } \\
\text { forecast*" or } \\
\text { forecast* }\end{array}$ & 548 & $\begin{array}{c}\text { Language: } \\
\text { English } \\
\text { Publication }\end{array}$ & 473 \\
\hline
\end{tabular}




\begin{tabular}{|c|c|c|c|c|}
\hline & technolog*" & & Stage: Final & \\
\hline $\begin{array}{c}\text { Technology } \\
\text { Foresight }\end{array}$ & $\begin{array}{l}\text { "technolog* } \\
\text { foresight*" or } \\
\text { foresight* } \\
\text { technolog*" }\end{array}$ & 205 & $\begin{array}{c}\text { Language: } \\
\text { English } \\
\text { Publication } \\
\text { Stage: Final }\end{array}$ & 188 \\
\hline
\end{tabular}

Fonte: Elaboração dos autores

Optou-se em não aplicar filtros no campo "Subject Areas", contemplando todas as áreas de estudo possíveis da temática. O objetivo dessa escolha foi identificar em quais áreas o termo é mais utilizado, podendo indicar, assim, o possível contexto de pesquisa em que a avaliação da tecnologia é feita.

Foram escolhidos os trabalhos pelo do título, palavras-chave, resumo e pela análise do RStudio em termos das redes de autores, número de citações e data. Buscou-se selecionar os artigos que na análise de redes construídas pelo software estivessem como nó principal, dando origem a um grande número de ramificações. Este critério foi adotado nos diferentes clusters de referências que foram formados. Ainda, foram escolhidos aqueles artigos com maior número de citações e com objetivo de captar os debates mais atuais, foi feito um balanceamento com trabalhos publicados recentemente. Foram excluídos os que não estavam disponíveis integralmente de forma gratuita, para acesso por meio da consulta pelo portal de periódicos da CAPES.

Por fim, referências extras, citadas por vários dos artigos selecionados, foram também coletadas e revisadas. Esses novos textos selecionados, foram aqueles que a partir do conteúdo citado e análise do título, demonstraram serem trabalhos relevantes para a caracterização da temática. Sendo assim, foram escolhidas as revisões de literatura, trabalhos que tinham como propósito analisar as visões de passado e futuro da área de conhecimento, e aqueles que se propunham a fazer estudos comparativos entre um ou mais termos que rementem à temática de avaliação de tecnologias e que estão sendo estudados no presente trabalho.

Os textos foram agrupados de acordo com as palavras chaves utilizadas durante a pesquisa. Inicialmente, o objetivo da análise era consolidar a escolha dos autores mais contributivos de cada área e entender as características das diferentes correntes de pesquisa. Por esse motivo, foram analisados os trabalhos de revisão, que traziam uma perspectiva histórica e da evolução das temáticas ao longo dos anos. A partir dessa leitura, caso algum trabalho relevante não tivesse sido considerado, este era incorporado na base de textos inicialmente consolidada. 
Após este passo inicial, buscou-se analisar os textos a partir dos seguintes elementos: contextos de aplicação da avaliação de tecnologia, principais objetivos do processo, principais agentes envolvidos na avaliação e quais são os principais métodos utilizados.

Os artigos de estudo de caso e as diferentes informações geradas pelo RStudio, como gráficos e tabelas foram usados na análise com intuito de auxiliar na construção dos argumentos para a caracterização das áreas relacionadas à cada palavra chave estudada.

\section{RESULTADOS E DISCUSSÃO}

Na sequência serão apresentados os resultados com as características principais de cada palavra chave pesquisada, bem como os principais métodos utilizados no processo de avaliação de tecnologias. Por fim, a partir da discussão será apresentada a tabela síntese com as considerações de cada uma das perspectivas.

\subsection{AVALIAÇÕES DE TECNOLOGIAS POR PROSPECÇÃO TECNOLÓGICA}

A análise dos textos evidenciou que uma das principais formas de se fazer avaliação de tecnologias é a partir dos estudos de prospecção tecnológica que analisam as perspectivas futuras da tecnologia ou área tecnológica que se deseja avaliar.

Segundo Porter et al. (2004), as análises futuras de tecnologias (do inglês Technology Future Analysis) representam qualquer processo sistemático para produzir julgamentos sobre características de tecnologias emergentes, seus caminhos de desenvolvimento e potenciais impactos no futuro. Essa visão mais ampla é uma denominação "guarda- chuva", construída a partir das correntes de pesquisa que se relacionam com os termos: "technology forecasting", "technology foresight" e "technology assessment".

Em muitos trabalhos foram observadas que essas diferentes terminologias são utilizadas para o mesmo fim. Mas ainda assim, é possível observar especificidades de conteúdo e variações do 
uso de cada termo ao longo dos anos. A Figura 1 demonstra o número de publicações por ano, de acordo com cada termo de busca pesquisado.

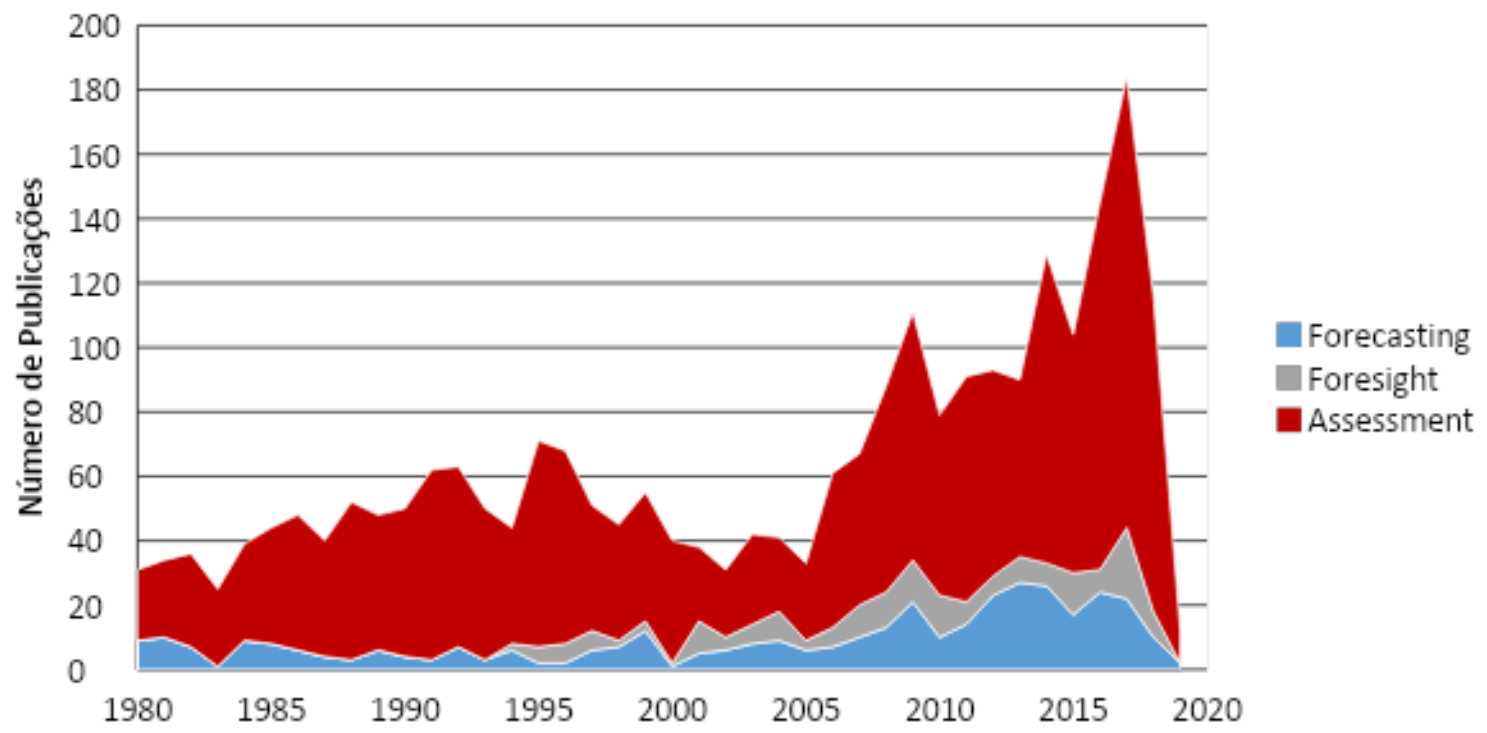

FIGURA 1 - Número de publicações por ano e por termo. Fonte: Elaboração dos autores

O termo Technology forecasting remete à estimativa do futuro de curto, médio ou longo prazo, por meio de metodologia científica, em uma área de pesquisa específica ou de acordo com as questões colocadas (CUHLS, 2003). Esse tipo de estudo, objetiva esclarecer em que direção o futuro da área selecionada pode evoluir. Martino (1993), um dos principais autores da área, afirma que um estudo na perspectiva do Technology Forecasting é composto por quatro elementos. O primeiro elemento está relacionado ao tempo considerado pelo estudo para a concretização das previsões. O segundo e o terceiro ponto estão relacionados à definição da tecnologia que está sendo estudada e suas principais características. E por fim é necessário que ocorra uma análise da probabilidade vinculada previsão realizada.

Já o Technology Foresight, segundo Martin (1996), refere-se a um processo sistemático de olhar para o futuro da ciência, tecnologia, economia e sociedade com o objetivo de identificar as áreas de pesquisa estratégicas e o surgimento de tecnologias capazes de produzir os maiores benefícios econômicos e sociais. Um dos trabalhos seminais na perspectiva de Technology Foresight é o de John Irvine e Ben Martin: seus livros Foresight in science, publicado em 1984 e Research Foresight em 1989. Segundo Miles (2010), esses autores passaram a utilizar 
métodos de análise do futuro na área de ciência e tecnologia e tiveram como principal objetivo nesses trabalhos entender como o governo priorizava seus investimentos para o financiamento de pesquisa.

A partir dessas definições, é perceptível a diferença entre Technology Forecasting e Technology Foresight. Enquanto, o primeiro busca entender melhor o futuro de uma temática específica, o segundo olha para o futuro de maneira mais ampla objetivando identificar as correntes tecnológicas que são mais promissoras. Por exemplo, na perspectiva de forecasting, o trabalho de Anderson et al (2008) aborda um estudo de tendências em comunicação wireless, tendo como foco uma área temática específica. De maneira distinta tem-se o trabalho de Martin et al (1999), demonstrando que as experiências em foresight de países como Reino Unido e Nova Zelândia não tiveram nenhuma especificidade em termos de área para a realização do estudo. Os programas desses países foram estruturados com uma característica holística e objetivos de definição de prioridades para desenvolvimento econômico.

Já o conceito de Technology Assessment, pode ser definido como um estudo desenhado para entender melhor as consequências secundárias da inserção de uma tecnologia, em termos de seus impactos sociais, culturais, políticos e ambientais (COATES, 2001). Este processo tem ênfase nos efeitos para a sociedade que normalmente não seriam planejados, ou seja, imprevistos. O foco em análise de impacto para a sociedade e seus aspectos de regulação tem sido expressado pelo grande número de trabalhos em periódicos relacionados a tecnologias da área da saúde. Destaca-se, na Tabela 2 a existência de periódicos específicos para publicações dessa natureza, comprovando a relação direta entre as temáticas.

TABELA 2 - Número de publicações de Technology Assessment por fonte.

\begin{tabular}{ll}
\hline \multicolumn{1}{c}{ Periódico } & $\mathbf{N}^{\mathbf{0}}$ de artigos \\
\hline International Journal of Technology Assessment in Health Care & 398 \\
Journal of the American Medical Association & 61 \\
Technological Forecasting and Social Change & 50 \\
Ontario Health Technology Assessment Series & 44 \\
Heathy Policy & 40 \\
Neurology & 36 \\
Value in Health & 33 \\
Clinical Governance: na International Journal & 21 \\
JAMA: the Journal of the American Medical Association & 20 \\
Science and Public Policy & 17 \\
International Journal of Technology Management & 16 \\
\hline
\end{tabular}

Fonte: Elaboração dos autores. 
Pelo seu escopo analítico e das amplas responsabilidades institucionais implícitas a visão de Technology Assessment é mais apropriadamente usada no setor público. De fato, a maior difusão deste viés de análise, aconteceu com a criação do OTA (Office of Technology Assessment) em 1972 nos Estados Unidos. Segundo Van Eijndhoven (1997) o escritório foi criado pelo congresso norte americano, para que, de maneira neutra, pudesse fornecer informações objetivas sobre os efeitos secundários da tecnologia, avaliando independentemente as virtudes dos desenvolvimentos tecnológicos para dar suporte na criação e alteração de políticas públicas. Ainda segundo o autor, a iniciativa inspirou a criação de outros escritórios com o mesmo propósito em países europeus como França, Holanda, Inglaterra, Alemanha e Dinamarca.

O poder público também está diretamente relacionado às abordagens de Technology Foresight, com uma tratativa diferente da de análise de impacto, mais voltada para a busca de desenvolvimento econômico da nação. Os governos podem utilizar estratégias de "Technology Foresight" como uma ferramenta para melhorar as redes e criar consenso nas comunidades de ciência e tecnologia ou em sistemas de inovação nacionais, regionais ou setoriais (PORTER et $a l, 2004)$. Trata-se, portanto, de uma ferramenta capaz de alertar os setores industriais sobre as diferentes oportunidades de desenvolvimento e demonstrar para a comunidade científica o significado social ou comercial e o potencial de seu trabalho.

Martin et al (1999), relatam as experiências dos programas desenvolvidos na Inglaterra, Austrália e Nova Zelândia. Grupp et al (1999), sumarizam a evolução da temática e, a partir das experiências de diversos países, destacam a atuação do Japão como referência.

A Figura 2 evidencia a produção científica dos países dentro da temática de Technology Foresight. É importante ressaltar a densidade de publicações de países que têm buscado acelerado desenvolvimento econômico nas últimas décadas impulsionado por crescente domínio tecnológico, como o caso da China, Coréia do Sul e Taiwan. 


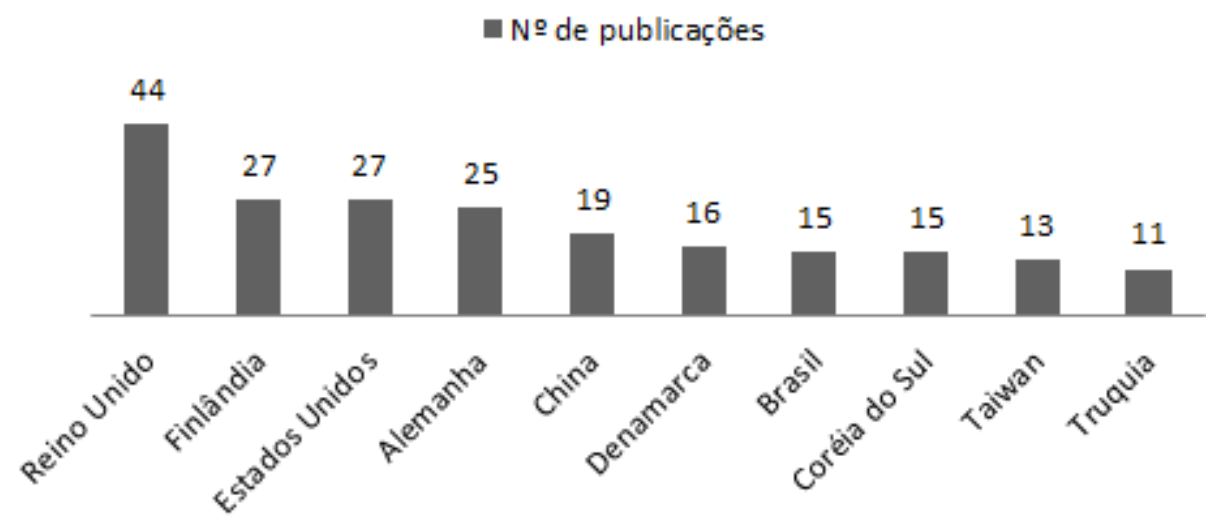

FIGURA 2 - Publicações por país relacionado à Technology Foresight. Fonte: Elaboração dos autores

Apesar de ter inicialmente as mesmas raízes da corrente do "Technology Assessment" as perspectivas de "Technology Forecasting” foram moldadas com o passar dos anos por uma ênfase na inovação, pelos interesses de mercado e pela crescente importância das indústrias intensivas em ciência.

Segundo Coates (2004), a partir dos anos 90, "Technology Forecasting” passou a ter como propósito principal, avaliar as ameaças e oportunidades relacionadas à inovação e seu efeito nos resultados financeiros da organização.

Pode-se dizer que tanto o governo quanto a indústria estão mais interessados no componente de exploração de mercado do desenvolvimento tecnológico. Mas há uma tendência geral no governo de usar frases que separam o pensamento da ação, como "assessment" e "foresight", enquanto na indústria há uma tendência a usar frases que ligam pensamento e ação, como "roadmapping" e "competitive technological intelligence" (COATES, 2004).

\subsection{MÉTODOS UTILIZADOS}

Apesar dos diferentes objetivos das perspectivas de avaliação de tecnologia, as análises dos artigos evidenciaram que de maneira geral existe uma convergência entre as metodologias utilizadas.

Porter et al (2004) fazem uma análise de 51 métodos utilizados em estudos das três temáticas de technology forecasting, foresight e assessment. Os métodos são qualificados em termos de família (criatividade; descritivo e matrizes; estatístico; opinião de especialistas; monitoramento e inteligência; modelagem e simulação; cenários; análise de tendências; valorização/decisão /econômico), em quantitativos ( $h a r d$ ) ou qualitativos (soft) e em normativos ou exploratórios. 
Alguns dos exemplos de métodos citados são: Método de Análise Hierárquica, Delphi, Análises Bibliométricas, Análise de Base de Patentes, Análise de Risco, Roadmapping, TRIZ, Análise de Impacto Social.

Dentro da perspectiva específica de forecasting, Martino (2002) ressalta em uma revisão teórica oito métodos e suas aplicações. São eles: Delphi, Extrapolação, Teoria do Caos, Previsões probabilísticas, Modelos Causais, Análise de Cenários e Monitoramento de Ambientes e Medição Tecnológica.

Já o trabalho de Tran et al (2008), apresenta uma taxonomia de métodos e ferramentas aplicados nos estudos de Technology Assessment. Os autores fazem a diferenciação entre os métodos e ferramentas utilizados no contexto do governo e aqueles utilizados por empresas e instituições não governamentais. Os autores concluem que no contexto formação de políticas públicas trabalha-se tipicamente empregando metodologias mais holísticas e multifacetadas como análises de impacto e de cenário, enquanto que o setor privado tende a utilizar métodos e ferramentas mais operacionais como roadmapping e análises de custo e benefício.

Um grande número de trabalhos tem sido desenvolvidos buscando a melhoria de resultados dos estudos de avaliação de tecnologias por meio do aprimoramento de aplicação de métodos. Parte dessa evolução representa melhorias nas técnicas existentes, no entanto, as inovações nos métodos estocásticos, por exemplo, representavam ferramentas antes indisponíveis para aqueles que atuam com análise de futuro de tecnologias (MARTINO, 2002).

Segundo Porter et al (2004), a maioria dos trabalhos de análises futuras de tecnologias utiliza diferentes métodos de análise, buscando balancear aqueles quantitativos e os qualitativos. E um dos desafios apresentados por (MISHRA et al, 2002 ) é a escolha dos métodos de acordo com a tecnologia o nível de informações disponível.

\subsection{SÍNTESES DAS PERESPECTIVAS ANALISADAS}

A partir do exposto no texto a Tabela 3 tem o objetivo de sumarizar a caracterização de cada perspectiva de avaliação de tecnologia analisadas. 
TABELA 3 - Caracterização das perspectivas de avaliação de tecnologias

\begin{tabular}{|c|c|c|c|c|}
\hline Palavra chave & Objetivos & $\begin{array}{l}\text { Contexto de } \\
\text { aplicação }\end{array}$ & $\begin{array}{c}\text { Principal ator } \\
\text { envolvido }\end{array}$ & $\begin{array}{c}\text { Principais } \\
\text { Referências }\end{array}$ \\
\hline $\begin{array}{l}\text { Technology } \\
\text { forecasting }\end{array}$ & $\begin{array}{c}\text { Identificar } \\
\text { oportunidades } \\
\text { em áreas } \\
\text { tecnológicas e } \\
\text { potencial de } \\
\text { tecnologias no } \\
\text { futuro. }\end{array}$ & $\begin{array}{c}\text { Tomada de } \\
\text { decisão em P\&D } \\
\text { e inteligência } \\
\text { competitiva nas } \\
\text { empresas }\end{array}$ & Empresas & $\begin{array}{c}\text { Porter et al } \\
\text { (2004); Martino } \\
\text { (1993); } \\
\text { Martino(2003); } \\
\text { Coates (2004). }\end{array}$ \\
\hline $\begin{array}{c}\text { Technology } \\
\text { Foresight }\end{array}$ & $\begin{array}{l}\text { Identificar e } \\
\text { avaliar } \\
\text { tendências no } \\
\text { contexto de } \\
\text { ciência } \\
\text { e tecnologia para } \\
\text { auxílio à tomada } \\
\text { de decisão. }\end{array}$ & $\begin{array}{c}\text { Criação de } \\
\text { planos de } \\
\text { desenvolvimento } \\
\text { tecnológicos } \\
\text { mais assertivos } \\
\text { para o } \\
\text { desenvolvimento } \\
\text { econômico do } \\
\text { país }\end{array}$ & Governo & $\begin{array}{l}\text { Porter et al } \\
\text { (2004); Martin } \\
\text { et al. (1999); } \\
\text { Miles (2010); } \\
\text { Cuhls (2003). }\end{array}$ \\
\hline $\begin{array}{l}\text { Technology } \\
\text { Assessment }\end{array}$ & $\begin{array}{c}\text { Antecipar } \\
\text { futuros impactos } \\
\text { na sociedade, } \\
\text { causados pela } \\
\text { inserção de } \\
\text { novas } \\
\text { tecnologias no } \\
\text { mercado ou pelo } \\
\text { aprimoramento } \\
\text { daquelas já } \\
\text { existentes. }\end{array}$ & $\begin{array}{c}\text { Criação de } \\
\text { políticas } \\
\text { públicas e de } \\
\text { regulação } \\
\text { principalmente } \\
\text { na área da saúde } \\
\text { e de meio } \\
\text { ambiente. }\end{array}$ & Governo & $\begin{array}{c}\text { Porter et al } \\
\text { (2004); Tran et al } \\
\text { (2008); Van } \\
\text { Eijndhoven } \\
\text { (1997); Coates } \\
\text { (2001). }\end{array}$ \\
\hline
\end{tabular}

Fonte: Elaboração dos autores.

\section{CONCLUSÃO}

O presente trabalho objetivou caracterizar as áreas de conhecimento relacionadas à "avaliação de tecnologias". Por meio de uma revisão sistemática foi possível identificar que a avaliação pode ser realizada a partir de análise do futuro dessas tecnologias. Historicamente, percebeu-se que os objetivos de se fazer a avaliação foram sendo alterados, saindo de uma visão de predição de impactos dessas tecnologias para a sociedade, no contexto de criação de políticas públicas, até como apoio a tomada de decisão no contexto de busca por desenvolvimento econômico seja do governo ou em empresas. Tal consideração reforça uma característica difusa da área de 
pesquisa, revelando que o processo de avaliação de tecnologias é sensível aos diferentes aspectos contingenciais.

De maneira de geral, os mesmos métodos são utilizados nas diferentes perspectivas de análise e muita das vezes os estudos são realizados a partir da aplicação de métodos com características opostas mas complementares. Os trabalhos mais recentes têm focado no aprimoramento desses métodos buscando cada vez mais adequá-los aos contextos de aplicação.

A partir desse embasamento, tem-se a oportunidade de em trabalhos futuros procurar entender como acontece o processo de avaliação de tecnologias nos diferentes contextos apontados, considerando aspectos organizacionais como disponibilidade recursos humanos e financeiros, tempo para a realização do estudo e principais entregas esperadas.

\section{REFERÊNCIAS}

ANDERSON, T. R.; DAIM, T. U.; KIM, Jisun. Technology forecasting for wireless communication. Technovation, v. 28, n. 9, p. 602-614, 2008.

BRERETON, P., KITCHENHAM, B. A., BUDGEN, D., TURNER, M., \& KHALIL, M. Lessons from applying the systematic literature review process within the software engineering domain. Journal of systems and software, 80(4), 571-583, 2007.

CASTELLS, M. Technopoles of the world: The making of 21st century industrial complexes. Routledge, 1995.

COATES, V. F. On the future of technological forecasting and assessment. International Journal of Foresight and Innovation Policy, v. 1, n. 3/4, p. 270, 2004.

COATES, J. F. A 21st Century Agenda for Technology Assessment. Technological Forecasting and Social Change, v. 67, n. 2-3, p. 303-308, 2001.

CUHLS, K. From forecasting to foresight processes - New participative foresight activities in Germany. Journal of Forecasting, v. 22, n. 2, p. 93-111, 2003.

GRUPP, H. LINSTONE, H. A. National technology foresight activities around the globe: resurrection and new paradigms. Technological Forecasting and social change, v. 60, n. 1, p. 85-94, 1999

KITCHENHAM, B. Procedures for performing systematic reviews. Keele, UK, Keele University, 33.2004: 1-26, 2004.

MARTIN, B. R. Technology Foresight Manual. Research Evaluation, v. 6, n. 2, p. 158-168, 1996.

MARTINO, J. P. A review of selected recent advances in technological forecasting. Technological forecasting and social change, v. 70, n. 8, p. 719-733, 2003.

MARTINO, J. P. Technological forecasting for decision making. McGraw-Hill, Inc., 1993.

MILES, I. The development of technology foresight: A review. Technological Forecasting and Social Change, v. 77, n. 9, p. 1448-1456, 2010.

MISHRA, S. DESHMUKH, S. G.; VRAT, Prem. Matching of technological forecasting technique to a technology. Technological Forecasting and Social Change, 69.1: 1-27, 2002.

PHAAL, R. Technology roadmapping - A planning framework for evolution and revolution. Technological Forecasting and Social Change, v. 71, n. 1-2, p. 5-26, 2004.

PORTER, A. L. et al. Technology futures analysis: Toward integration of the field and new methods. 
11 a 13 de setembro de 2019 - Universidade de Brasília UnB

Technological Forecasting and Social Change, v. 71, n. 3, p. 287-303, 2004.

Tran, T. A., \& Daim, T. (2008). A taxonomic review of methods and tools applied in technology assessment. Technological Forecasting and Social Change, 75(9), 1396-1405.

Van Eijndhoven (1997) Technology assessment: Product or process? Technological Forecasting and Social Change 54 (1997) 269-286. 